\title{
Improving Growth and Seed Yield of Squash by Foliar Applications with Moringa Leaf Extract, Ascorbic Acid or Benzyladenine
}

\author{
A. Z. Hegazi, A. Y. Ismaiel and T. G. Anany \\ Vegetable Crops Seed Production and Technology Department, \\ Horticulture Research Institute, Agriculture Research Centre, \\ Cairo, Egypt.
}

$\mathbf{F}$ OLIAR SPRAY with nutrients and other amendments is becoming an essential addition to standard cultivation techniques and must be adjusted carefully to avoid off-target spray. Thus, this work has been intended to study the effect of foliar application of squash plants (var. Eskandrani) with moringa (Mo) dry leaf water extract $(2,4$ and $6 \mathrm{~g} / \mathrm{L})$, cytokines as benzyladenine (BA) or ascorbic acid (AsA) (50,100,200 ppm for both) and its reflect on the growth and seed yield. For that, two field experiments were conducted during summer seasons of 2013 and 2014 at Kaha, Kalyiobia Governorate, Horticulture Research Station, Egypt. Most examined growth attributes responded positively and gave significant increase than the control by adding the previous compounds. Concerning to moringa; the positive effect was directly proportional to the concentration used, while in BA and AA; it is clear that 100 ppm was the best concentration.

Squash plants sprayed with BA at $100 \mathrm{ppm}$ recorded the highest mean value for number of leaves/ plant, leaves area/ plant and seed yield. Similarly, using AsA at $100 \mathrm{ppm}$ had positive significant effect for plant height and fresh \& dry weight/ plant. Also, the germination rate, i.e. the longevity of the period that the seeds required to indicate obvious germination, showed a significant decrease from the control by using any type or concentration of the applied substances.

All the compounds with its concentrations induced enhancement effect on NPK seed content compared to the control. In addition, BA $100 \mathrm{ppm}$ appeared to represent the best stimulus for plant chlorophyll with a concomitant enhancement in carbohydrates content.

The results revealed that foliar application of squash plants with moringa leaf extract at the higher dose $6 \mathrm{~g} / \mathrm{L}$ or benzyladenine as well as ascorbic acid at $100 \mathrm{ppm}$ significantly increased the growth and seed yield parameters over the control with a concomitant enhancement on NPK seed content, leaves chlorophyll and carbohydrates.

Keywords: Squash, Moringa leaf extract, Ascorbic acid, Benzyladenine, growth, Seed yield. 
All plants can absorb nutrients through their leaves and stems, thus foliar spraying is a valuable way to give the plants a varied and balanced diet by applying any needs or deficient compounds. Amendment kinds or concentrations must be selected and adjusted carefully to avoid off-target spray. Vegetables which include squash can be sprayed to hasten and regulate plant metabolism to improve its growth. Squash fruit contain more than $95 \%$ water, low in calories and a good source for vitamin C. Squash seeds are rich source for protein, oil and valuable active ingredients such as fatty acids, phytosterols and vitamin $\mathrm{E}$ (El Kramany et al., 2007).

Moringa is one of the most useful trees and one of the promising plants as almost every part of the tree has some beneficial properties and can be used in different purposes (Bamishaiye et al., 2011). Moringa leaves are rich in many active ingredients such as hormones and cytokines (Duck, 1992), thus its extract is considered natural stimulative substances for plants and enhance yield when applied as foliar spray. Moringa leaf extract was sprayed onto leaves of melon, onions, bell pepper, soya beans, and chili and induced obvious increases on the yields of these crops (Fuglie, 2000). In another trial (Culver et al., 2012) reported that moringa extract significantly increased above ground dry matter yield, root dry matter weight and plant height for tomato in both greenhouses and open field. Sweet pepper plants treated with $80 \mathrm{~g} / \mathrm{L}$ of moringa leaf powder per plot recorded the highest mean value of plant height, number of leaves, number of fruits per plant, fruit weight per plant, total yield per plot and the thickest plant girth (Sowley et al., 2014).

Cytokinine such as Benzyladenine (BA), zeiaten and kinetine are growth regulators. Physiological role of this group of plant hormones is connected with stimulating cell divisions, delaying ageing processes, stimulating seed germination, and regulation of biochemical processes (Borkowska, 1997). Applied $500 \mathrm{mg} / \mathrm{l}$ kinetin to lentil showed insignificant increase in its growth as compared to control in the number of compound leaves and leaves area which being $14.72 \%$ after 30 days (Naeem et al. 2004). BA alone or in combination with gibberellic acid has the potential to be used to develop the growth of cucumber plants in terms of fruit size and yield (Batlang et al., 2006).

Another foliar spray such as ascorbic acid (AsA) which considered the most abundant water-soluble antioxidant in plants (Suza et al., 2010), can be used for improving vegetable performance. Foliar application of AsA resulted in a significant increment of vegetative growth (including plant height, number of leaves, number of branches and fresh weight of plants) and yield of eggplant compared to control plants (El-Tohamy et al., 2008). Foliar application of (AsA) induced a stimulatory effect on growth parameters of kidney bean which were accompanied by marked increases in nutrients concentration and photosynthetic system. In addition AsA application may induce an adaptive response in bean through stimulation of the antioxidant enzymes activities, photosynthesis processes and lower lipid peroxidation (Salama et al., 2014).

Egypt. J. Hort. Vol. 42, No.1 (2015) 
The objectives of this study were to evaluate the effects of foliar application with moringa leaves extract, benzyladenine or ascorbic acid on growth and seed yield of squash under open field condition.

\section{Material and Methods}

Pure seeds lot of squash (Cucurbita pepo L.) variety Eskandrany was obtained from the Vegetable Crops Seed Production and Technology Department, Horticulture Research Institute, Agriculture Research Center, Egypt. Two open field experiments were conducted during summer seasons of 2013 and 2014 at Kaha (Kalyiobia Governorate), Horticulture Research Station, Egypt. The experiment was carried out to evaluate the influence of different foliar spray substances, i.e. moringa leaf water extract or Bezyleadenine (BA) or ascorbic acid (AsA) on growth, seed yield and seed quality of squash. Moringa leaf extract was applied at dose of 2, 4, $6 \mathrm{~g} / \mathrm{L}$, while BA or AsA were used at levels 50, 100, $200 \mathrm{ppm}$. The experiments were laid out in a randomized complete block design with three replications. The foliar spray was carried out three times; the first application was applied after 4 weeks from sowing and then two times two weeks intervals. Sowing was done on $1^{\text {st }}$ week of March during the two successive seasons of the present work. Each treatment containe 3 rows $4 \mathrm{~m}$ long and $0.80 \mathrm{~m}$ wide, making an area of $9.6 \mathrm{~m}^{2}$. Hills were done $40 \mathrm{~cm}$ apart; 2 seeds per hill then thinned three weeks later to 1 plant/ hill. Other cultural practices were carried out as recommended for the conventional squash planting.

The substances used in this study were prepared as following

Moringa fresh leaves were picked and spread on dry blotters and left three days for to reach complete air dryness. During this period, leaves are bowed regularly to avoid fungal growth or rotting. The air dried leaves were grinded and sieved to become powder. This powder was homogenizes with water in blender according to the required concentration used, i.e. 2,4 or $6 \mathrm{~g} / \mathrm{L}$, then filtrated throw cotton cloth.

Ascorbic acid and benzyladenine were obtained as commercial chemical substances from El-Gomhorya Company (Egypt).

\section{Measurements \\ Vegetative growth characters}

The following growth attributes were measured at the end of flowering stage, using ten random plants from each plot: plant height $(\mathrm{cm})$, number of branches/plant, number of leaves/plant, plant fresh weight $(\mathrm{g})$ and plant dry weight $(\mathrm{g})$.

\section{Seed yield and its components}

Squash fruits at seed stage were harvested for seed extraction. A sample of five random fruits from each replicates was used to record the following characters: seed weight/plant $(\mathrm{g})$ and from seed weight per plot total seed yield 
(kg/fed.) can be calculated. Almost, one fruit per plant could be mature for seed yield, while other fruits became atrophied.

\section{Chemical constitute}

At the end of flowering stage (about 50 days from sowing), top fifth leaf from 3 random plants in each of the three replicates were taken for determination of chlorophyll using Minolta chlorophyll meter SPAD- 502 as SPAD units. Total carbohydrates (\%) were colorimetrically determined in leaves according to Herbert et al. (1971). Minerals (N P K) were determined as $\%$ in dry seeds according to (AOAC, 2003).

\section{Seed quality}

Three random samples of 100 seeds from each treatment were used for calculating the following records; weight of 100 seeds $(\mathrm{g})$, germination percentage $(\%)$, germination rate, seedling length $(\mathrm{cm})$. Germination rate was calculated according to the following equation;

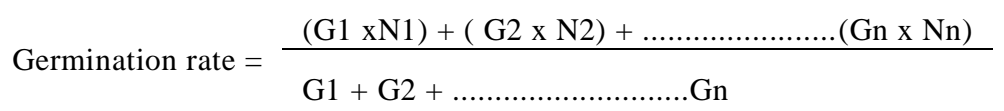

Where: $\mathrm{G}=$ Number of germinated seeds in a certain day, $\mathrm{N}=$ Number of this certain day

\section{Statistical analysis}

All the collected data were tabulated and statistically analyzed using the analyses of variance method as reported by Snedecor and Cochran (1980) and the treatments means were compared using the Duncan Multiple Range test (Duncan, 1955).

\section{Vegetative parameters}

\section{Results and Discussion}

Vegetative growth parameters as shown in Table 1 indicated that all substances used as foliar spray gave better results than the control. Cytokine at $100 \mathrm{ppm}$ gave the best significant results in both seasons for no. of leaves/ plant, leaf area/ plant and dry weight/ plant. Ascorbic acid at $100 \mathrm{ppm}$ recorded the best significant results in both season for fresh weight/ plant and for plant height in second season only, while in the first season moringa leaf extract $(6 \mathrm{~g} / \mathrm{L})$ recorded the best significant increase for that criterion.

In this connection, Naeem et al. (2004) detected improvement in vegetative growth of lentil indicated by leaf expansion and increase in leaves number and their area by applied kinetin, as a type of cytokine, at dose of 30 $\mathrm{m} \mathrm{g} / \mathrm{L}$. Also, application of ascorbic acid as a foliar spray showed an increment in growth attributes of mung bean plants (Chaudhary \& Agrawal 2014). 
IMPROVING GROWTH AND SEED YIELD OF SQUASH...

\begin{tabular}{|c|c|c|c|c|c|c|c|c|c|c|c|}
\hline \multirow{5}{*}{ 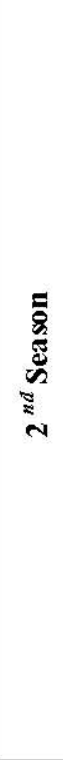 } & 昜高 &  & $\begin{array}{l}0 \\
0 \\
\infty \\
\infty \\
\tilde{q}\end{array}$ & 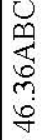 & $\begin{array}{l}\text { a } \\
\text { d } \\
0 \\
0 \\
0 \\
\sigma\end{array}$ & 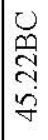 & $\begin{array}{l}\varangle \\
8 \\
8 \\
8\end{array}$ & 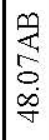 & 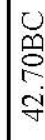 & $\begin{array}{l}0 \\
0 \\
0 \\
0 \\
y \\
y\end{array}$ & 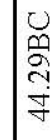 \\
\hline & 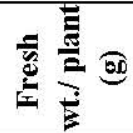 & $\begin{array}{l}0 \\
0 \\
\text { ci } \\
\text { d }\end{array}$ & 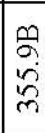 & $\begin{array}{l}0 \\
0 \\
0 \\
0 \\
0 \\
0\end{array}$ & $\begin{array}{l}q \\
\infty \\
\infty \\
\infty \\
\infty\end{array}$ & $\begin{array}{l}\stackrel{p}{2} \\
\text { di } \\
\text { r }\end{array}$ & $\begin{array}{l}m \\
0 \\
i \\
\text { in } \\
m\end{array}$ & $\begin{array}{l}\stackrel{9}{Z} \\
\dot{0} \\
\text { d }\end{array}$ & 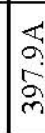 & & 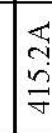 \\
\hline & 离 & 踈 & 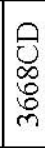 & 己ृ & $\begin{array}{l}0 \\
0 \\
0 \\
\infty \\
m\end{array}$ & $\begin{array}{l}\text { 됨 } \\
\text { 을 } \\
\text { ․ } \\
\text { m }\end{array}$ & $\mid \begin{array}{c}\frac{5}{5} \\
\frac{\delta}{\sigma} \\
\end{array}$ & 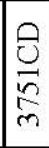 & $\begin{array}{l}0 \\
0 \\
\infty \\
m \\
m\end{array}$ & & $\frac{\mathscr{m}}{\stackrel{?}{f}}$ \\
\hline & 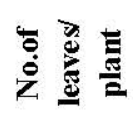 & $\begin{array}{l}0 \\
8 \\
i \\
i \\
-\end{array}$ & $\begin{array}{l}0 \\
0 \\
0 \\
0 \\
0 \\
-0\end{array}$ & 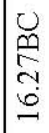 & $\begin{array}{l}\text { 点 } \\
\stackrel{\Xi}{\Xi} \\
=\end{array}$ & 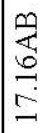 & $\underset{m}{\stackrel{\mathbb{m}}{\omega}}$ & 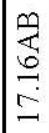 & $\begin{array}{l}0 \\
0 \\
0 \\
0 \\
0\end{array}$ & & 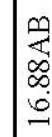 \\
\hline & 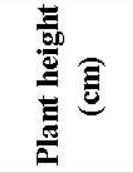 & $\begin{array}{l}8 \\
\text { ळे } \\
\text { Һे }\end{array}$ & $\begin{array}{l}8 \\
m \\
m \\
m \\
\bar{n}\end{array}$ & 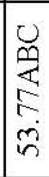 &  & $\begin{array}{l}80 \\
8 \\
n \\
i n \\
i n\end{array}$ & 空 & $\begin{array}{l}0 \\
0 \\
\sum_{n} \\
n \\
\tilde{n}\end{array}$ & 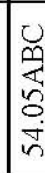 & & 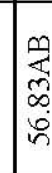 \\
\hline \multirow{5}{*}{ 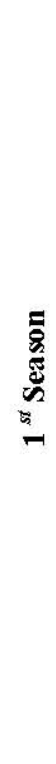 } & 昜 & $\underset{\substack{n \\
\text { m. }}}{m}$ & $\begin{array}{l}a \\
\infty \\
\infty \\
m \\
m\end{array}$ & 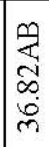 & 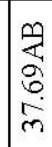 & 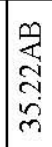 & $\begin{array}{l}\varangle \\
\delta \\
\delta \\
\sigma\end{array}$ & $\begin{array}{l}\frac{m}{2} \\
\vdots \\
0 \\
\infty \\
\text { m. }\end{array}$ & 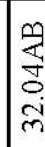 & $\begin{array}{l}\frac{m}{5} \\
\frac{7}{2}\end{array}$ & $\begin{array}{l}\text { ầ } \\
\text { ç } \\
\text { ले }\end{array}$ \\
\hline & 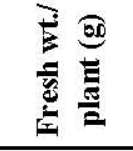 & $\begin{array}{l}0 \\
0 \\
\text { ì } \\
\text { ते }\end{array}$ & $\begin{array}{l}0 \\
0 \\
o \\
i \\
\sim \\
m\end{array}$ & 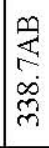 & $\begin{array}{l}\text { m } \\
\infty \\
\text { in } \\
\text { ñ }\end{array}$ & $\begin{array}{l}\frac{m}{2} \\
\text { ci } \\
\text { ले }\end{array}$ & $\begin{array}{l}\text { qu } \\
\text { d } \\
\text { jं } \\
\text { mे }\end{array}$ & $\begin{array}{l}\text { 是 } \\
\text { o } \\
\dot{\dot{m}} \\
\text { m }\end{array}$ & $\begin{array}{l}n \\
\vdots \\
\vdots \\
0 \\
0 \\
0\end{array}$ & 疍 & $\begin{array}{l}\mathbb{N} \\
\text { w } \\
\infty \\
m\end{array}$ \\
\hline & 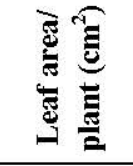 & 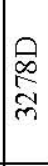 & $\begin{array}{l}0 \\
\infty \\
\infty \\
0 \\
0\end{array}$ & $\begin{array}{l}0 \\
0 \\
0 \\
0 \\
0 \\
0 \\
\text { o }\end{array}$ & 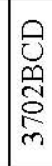 & $\begin{array}{l}\stackrel{8}{8} \\
\text { के } \\
\text { m. }\end{array}$ & $\mid \begin{array}{l}\text { 志 } \\
\text { 索 }\end{array}$ & 尝 & $\begin{array}{l}0 \\
0 \\
0 \\
\infty \\
m \\
m\end{array}$ & & 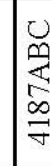 \\
\hline & 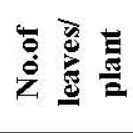 & $\begin{array}{l}\text { m} \\
8 \\
\text { i }\end{array}$ & 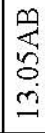 & 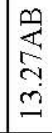 & 亲 & 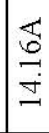 & 芯 & $\begin{array}{l}\frac{m}{4} \\
8 \\
\dot{8} \\
\dot{m}\end{array}$ & $\begin{array}{l}\frac{9}{4} \\
0 \\
m \\
m\end{array}$ & 要 & $\begin{array}{l}\overleftrightarrow{\infty} \\
\infty \\
\stackrel{\infty}{-}\end{array}$ \\
\hline & 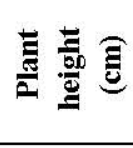 & 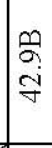 & $\begin{array}{l}\frac{9}{2} \\
\frac{1}{2} \\
\frac{8}{n}\end{array}$ & 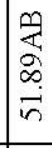 & 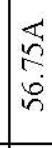 & 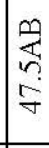 & 学 & $\begin{array}{l}\text { 䍃 } \\
\text { = } \\
\text { in }\end{array}$ & $\begin{array}{l}m \\
\text { ât } \\
\text { ले } \\
\text { in }\end{array}$ & & $\mid \begin{array}{l}\infty \\
\infty \\
\end{array}$ \\
\hline & & $\bar{\delta}$ & $\overrightarrow{0}$ & $\sum^{\delta}$ & $\sum_{\Sigma}^{0}$ & $\begin{array}{l}\overrightarrow{0} \\
\text { 热 } \\
\text { d }\end{array}$ & 志 & 商 & 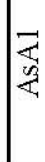 & & $\mid \begin{array}{l}3 \\
\substack{3 \\
\frac{1}{4}}\end{array}$ \\
\hline
\end{tabular}

Egypt. J. Hort. Vol. 42, No.1 (2015) 
Seed yield

The results obtained (Table 2) showed that seed yiel/ plant and seed yield/ fed. were generally increased as a result of moringa application in a magnitude that was directly proportional to the concentration used. However, such effect was changed in case of using cytokines as benzyladenine or ascorbic acid where the increment continued gradually up to the second concentration (100 ppm) used then decrease with the third concentration $(200 \mathrm{ppm})$ but it still higher than the control for each. The increments in seed yield/fed with benzyladenine application at $100 \mathrm{ppm}$ was $50.9 \%$ and $47.7 \%$ over the control in first and second seasons, respectively. Thus we can use concentration more than 100 ppm but less than 200 ppm to give better results in case of using benzyladenine as well as ascorbic acid. It is also clear from the same table that the increase in squash seed yield/ fed was due to the increases of the other two parameters i.e. seed index (wt of 100 seeds) and seed yield/ plant. This trend was similar in both seasons of the experiment. Several invistigators explained the important of role of suing the mentioned substances for improving the crops yield. Moringa leaves is enrich with cytokinin, antioxidants and minerals (Azra et al., 2014) and thus its extract application improved productivity of tomato. Also application of Accel (a type of cytokines) increased number of snap bean pods per plant and pod yield per hectare (Emongor, 2007). In addition, Ibrahim et al. (2007) detected marked increase in vicia seed yield (pod length, number of pods / plant, number and weight of seeds per pod as well as weight of 100 seeds) from the application of benzyl adenine as bioregulators at a rate of $100 \mathrm{ppm}$.

TABLE 2. Seed yield and seed index of squash as affected by foliar application with different concentrations of moringa leaves extract (Mo), cytokinene (cyto) or ascorbic acid (Asc) in $2013 \& 2014$ seasons.

\begin{tabular}{|c|c|c|c|c|c|c|}
\hline \multirow{2}{*}{ Treatment $^{\mathrm{z}}$ Character } & \multicolumn{3}{|c|}{$1^{\text {st }}$ Season } & \multicolumn{3}{|c|}{$2^{\text {nd }}$ Season } \\
\hline & $\begin{array}{c}\text { Seed index } \\
\text { (wt of } 100 \\
(\mathrm{~g}))\end{array}$ & $\begin{array}{c}\text { Seed } \\
\text { yield/ } \\
\text { plant (g) }\end{array}$ & $\begin{array}{c}\text { Seed } \\
\text { yield } \\
\text { /fed. (kg) }\end{array}$ & $\begin{array}{c}\text { Seed } \\
\text { index } \\
\text { (wt of } \\
100(g))\end{array}$ & $\begin{array}{c}\text { Seed yield/ } \\
\text { plant (g) }\end{array}$ & $\begin{array}{c}\text { Seed } \\
\text { yield /fed } \\
(\mathbf{k g})\end{array}$ \\
\hline Cont. & $10.93 \mathrm{C}$ & $28.98 \mathrm{CD}$ & $221.8 \mathrm{E}$ & $15.93 \mathrm{C}$ & $38.97 \mathrm{C}$ & $209.1 \mathrm{E}$ \\
\hline Mo1 & $14.50 \mathrm{AB}$ & $32.22 \mathrm{BC}$ & $266.6 \mathrm{D}$ & $19.49 \mathrm{AB}$ & $42.25 \mathrm{BC}$ & $278.0 \mathrm{D}$ \\
\hline Mo2 & $15.47 \mathrm{AB}$ & $33.61 \mathrm{~B}$ & $286.7 \mathrm{CD}$ & $21.70 \mathrm{~A}$ & $43.61 \mathrm{ABC}$ & $284.7 \mathrm{CD}$ \\
\hline Mo3 & $15.17 \mathrm{AB}$ & $34.92 \mathrm{~B}$ & 294.4 BC & $20.84 \mathrm{AB}$ & $42.36 \mathrm{BC}$ & 297.2 BCD \\
\hline Cyto1 & $14.45 \mathrm{AB}$ & $35.19 \mathrm{~B}$ & $299.1 \mathrm{BC}$ & $19.45 \mathrm{AB}$ & $45.19 \mathrm{AB}$ & $299.0 \mathrm{BC}$ \\
\hline Cyto2 & $16.78 \mathrm{~A}$ & $39.02 \mathrm{~A}$ & $334.6 \mathrm{~A}$ & $21.80 \mathrm{~A}$ & $49.02 \mathrm{~A}$ & $338.3 \mathrm{~A}$ \\
\hline Cyto3 & $15.11 \mathrm{AB}$ & $35.32 \mathrm{~B}$ & $311.6 \mathrm{AB}$ & $20.11 \mathrm{AB}$ & $45.32 \mathrm{AB}$ & $314.3 \mathrm{~B}$ \\
\hline AsA1 & $13.13 \mathrm{BC}$ & $27.20 \mathrm{D}$ & $290.0 \mathrm{BCD}$ & $18.13 \mathrm{BC}$ & $40.53 \mathrm{BC}$ & 296.8 BCD \\
\hline AsA2 & $15.86 \mathrm{AB}$ & $29.96 \mathrm{CD}$ & $302.3 \mathrm{BC}$ & $20.86 \mathrm{AB}$ & $42.62 \mathrm{BC}$ & $301.2 \mathrm{BC}$ \\
\hline AsA3 & $13.66 \mathrm{BC}$ & $29.31 \mathrm{CD}$ & $284.7 \mathrm{CD}$ & $18.65 \mathrm{BC}$ & $42.64 \mathrm{BC}$ & $302.8 \mathrm{BC}$ \\
\hline
\end{tabular}

${ }^{2}$ :Mo 1,2,3=2,4,6 (g/l), respectively; Cyto 1,2,3= 50,100,200 (ppm), respectively; AsA 1,2,3= 50, $100,200(\mathrm{~g} / \mathrm{l})$, respectively.

Values within the same column followed by the same letters are not significantly different at $5 \%$ level Duncan's multiple range test.

Egypt. J. Hort. Vol. 42, No.1 (2015) 


\section{Seed quality}

Data represented in Table 3 showed a significant increase than the control in germination percentage using benzyladenine $100 \mathrm{ppm}$ in both seasons and when using ascorbic acid $100 \mathrm{ppm}$ in second season only. Seedling length increased significantly in first season with the applications: cyto2, cyto3, AsA1 and AsA2 and in second season with the applications: cyto2 and AsA2. On the other hand, germination rate, i.e. the longevity of the period that the seeds required to indicate obvious germination (appearance of radical), showed significant decrease from the control by using the substances in this study with superiority by using second cytokine concentration. It means that the seeds produced from plants which received any type or concentrations of applied substances germinate faster than the control.

In a study carried out by Phiri and Mbewe (2010) on three common legumes (beans, cowpea and groundnut), authors indicated that moringa leaves extract (rich in cytokine as mentioned previously) forced beans to germinate early, increased germination percentage of cowpea by $4 \%$, increased radical length by $4 \%$ in beans and increased hypocotyl length by $16.6 \%$ in groundnut. Also ascorbic acid has been reported to promote germination of seeds (Bauernfeind, 2009). In Eruca sativa, germination percentage, speed of germination, mean germination time as well shoot and root length were significantly affected by ascorbic acid treatment (Jyoti and Malik, 2013).

TABLE 3. Germination percentage (\%), germination rate and seedling length of squash as affected by foliar application with different concentrations of moringa leaves extract (Mo), cytokinene (cyto) or ascorbic acid (Asc) in 2013\&2014 seasons.

\begin{tabular}{|c|c|c|c|c|c|c|}
\hline \multirow{2}{*}{ Character } & \multicolumn{3}{|c|}{$1^{\text {st }}$ Season } & \multicolumn{3}{c|}{$2^{\text {nd }}$ Season } \\
\cline { 2 - 7 } & $\begin{array}{c}\text { Germination } \\
(\%)\end{array}$ & $\begin{array}{c}\text { Germination } \\
\text { rate (day) }\end{array}$ & $\begin{array}{c}\text { Seedling } \\
\text { length } \\
(\mathbf{c m})\end{array}$ & $\begin{array}{c}\text { Germination } \\
\%\end{array}$ & $\begin{array}{c}\text { Germination } \\
\text { rate (day) }\end{array}$ & $\begin{array}{c}\text { Seedling } \\
\text { length } \\
(\mathbf{c m})\end{array}$ \\
Treatment \\
\hline CONT. & $95.30 \mathrm{BC}$ & $3.70 \mathrm{~A}$ & $21.31 \mathrm{~B}$ & $94.00 \mathrm{~B}$ & $3.85 \mathrm{~A}$ & $21.87 \mathrm{~B}$ \\
\hline Mo1 & $97.00 \mathrm{BC}$ & $3.37 \mathrm{BC}$ & $21.75 \mathrm{~B}$ & $94.80 \mathrm{~B}$ & $3.44 \mathrm{BC}$ & $22.87 \mathrm{AB}$ \\
\hline Mo2 & $97.75 \mathrm{AB}$ & $3.18 \mathrm{DE}$ & $22.00 \mathrm{~B}$ & $95.00 \mathrm{~B}$ & $3.34 \mathrm{BCD}$ & $23.00 \mathrm{AB}$ \\
\hline Mo3 & $98.00 \mathrm{AB}$ & $3.14 \mathrm{DE}$ & $22.50 \mathrm{~B}$ & $96.00 \mathrm{AB}$ & $3.22 \mathrm{BCD}$ & $24.25 \mathrm{~A}$ \\
\hline Cyto1 & $98.00 \mathrm{AB}$ & $3.24 \mathrm{CD}$ & $22.75 \mathrm{~B}$ & $96.00 \mathrm{AB}$ & $3.50 \mathrm{~B}$ & $22.91 \mathrm{AB}$ \\
\hline Cyto2 & $100.00 \mathrm{~A}$ & $3.06 \mathrm{E}$ & $28.25 \mathrm{~A}$ & $98.00 \mathrm{~A}$ & $3.06 \mathrm{D}$ & $24.62 \mathrm{~A}$ \\
\hline Cyto3 & $96.67 \mathrm{BC}$ & $3.17 \mathrm{DE}$ & $26.55 \mathrm{~A}$ & $96.00 \mathrm{AB}$ & $3.18 \mathrm{BCD}$ & $23.06 \mathrm{AB}$ \\
\hline AsA1 & $96.67 \mathrm{BC}$ & $3.39 \mathrm{~B}$ & $26.62 \mathrm{~A}$ & $94.00 \mathrm{~B}$ & $3.32 \mathrm{BCD}$ & $23.60 \mathrm{AB}$ \\
\hline AsA2 & $97.60 \mathrm{BC}$ & $3.08 \mathrm{E}$ & $27.75 \mathrm{~A}$ & $97.33 \mathrm{~A}$ & $3.16 \mathrm{CD}$ & $23.90 \mathrm{AB}$ \\
\hline AsA3 & $96.00 \mathrm{BC}$ & $3.46 \mathrm{~B}$ & $23.25 \mathrm{~B}$ & $96.33 \mathrm{AB}$ & $3.16 \mathrm{CD}$ & $23.25 \mathrm{BA}$ \\
\hline
\end{tabular}

$\mathrm{z}$ :Mo 1,2,3= 2,4,6 (g/l), respectively; Cyto 1,2,3=50,100,200 (ppm), respectively; AsA 1,2,3= 50, $100,200(\mathrm{~g} / \mathrm{l})$, respectively.

Values within the same column followed by the same letters are not significantly different at $5 \%$ level Duncan's multiple range test. 
Chemical composition of seeds

It is evident from Table 4 that the contents of squash seeds from N P K showed an increase by using various spray concentrations. In the first season, the best value for $\mathrm{N}$ was obtained from Asc at $200 \mathrm{ppm}$, for $\mathrm{P}$ from Mo $6 \mathrm{~g} / \mathrm{L}$ while for $\mathrm{K}$ from Asc at $100 \mathrm{ppm}$. In second season there is a slight change whereas the best results for $\mathrm{N}$ was indicated by using Mo3, for $\mathrm{P}$ by using cyto 2 and for $\mathrm{K}$ similar like the trend in first season. All above mentioned concentrations gave significant increase than the control. Also the data in same table indicated that there is a concomitant increase in $\mathrm{N} \mathrm{P} \mathrm{K}$ as moringa extract concentration increased. Cyto at a dose 100 ppm was the best for $\mathrm{N}$ and $\mathrm{P}$ and cyto 3 for $\mathrm{K}$. In case of ascorbic acid application, the best results for $\mathrm{N}$ were obtained with Asc3 while for P and $\mathrm{K}$ with Asc2. In this study; NPK enhancement by using different foliar spray may be due to those applications gave turgidity to plant cells and hence decrease solute leakage and also protect the plants from environmental injury. In this connection, El-Tohamy et al. (2008) and El-Hifny and El-Sayed (2011) detected an increase in N,P and $\mathrm{K}$ in sweet pepper plant leaves when foliar spray of ascorbic acid at the highest level (400 mg/L) combined with some biofertilizers had been applied. Also, mineral ions $(\mathrm{Ca}, \mathrm{K}$, and $\mathrm{Mg}$ ) contents of Vicia faba L. plants were increased due to foliar application of benzyl adenine at the rate of 100 ppm (Ibrahim et al., 2007).

TABLE 4. $\mathbf{N}, \mathbf{P}$, and $\mathrm{K}$ percentage in squash seeds as affected by foliar application with different concentrations of moringa leaves extract (Mo), cytokinene (cyto) or ascorbic acid (Asc) in 2013\&2014 seasons.

\begin{tabular}{|l|c|c|c|c|c|c|}
\hline \multirow{2}{*}{} & \multicolumn{3}{|c|}{$1^{\text {st }}$ Season } & \multicolumn{3}{c|}{$2^{\text {nd }}$ Season } \\
\cline { 2 - 7 } & & & & & & \\
Treatment & & & & & \\
\hline CONT. & $4.00 \mathrm{D}$ & $1.03 \mathrm{G}$ & $0.78 \mathrm{~F}$ & $3.72 \mathrm{DE}$ & $1.04 \mathrm{G}$ & $0.76 \mathrm{E}$ \\
\hline Mo1 & $4.41 \mathrm{AB}$ & $1.34 \mathrm{CD}$ & $0.80 \mathrm{~F}$ & $3.86 \mathrm{CD}$ & $1.20 \mathrm{~F}$ & $0.78 \mathrm{DE}$ \\
\hline Mo2 & $4.42 \mathrm{AB}$ & $1.80 \mathrm{~B}$ & $0.84 \mathrm{E}$ & $4.38 \mathrm{AB}$ & $1.32 \mathrm{E}$ & $0.80 \mathrm{CD}$ \\
\hline Mo3 & $4.53 \mathrm{~A}$ & $1.96 \mathrm{~A}$ & $0.88 \mathrm{CD}$ & $4.44 \mathrm{~A}$ & $1.61 \mathrm{~B}$ & $0.84 \mathrm{~B}$ \\
\hline Cyto1 & $4.13 \mathrm{CD}$ & $1.12 \mathrm{~F}$ & $0.84 \mathrm{E}$ & $3.70 \mathrm{DE}$ & $1.41 \mathrm{D}$ & $0.78 \mathrm{DE}$ \\
\hline Cyto2 & $4.54 \mathrm{~A}$ & $1.4 \mathrm{C}$ & $0.86 \mathrm{DE}$ & $4.21 \mathrm{~B}$ & $1.94 \mathrm{~A}$ & $0.78 \mathrm{DE}$ \\
\hline Cyto3 & $4.23 \mathrm{BC}$ & $1.21 \mathrm{E}$ & $0.90 \mathrm{BC}$ & $4.00 \mathrm{C}$ & $1.61 \mathrm{~B}$ & $0.82 \mathrm{BC}$ \\
\hline AsA1 & $4.10 \mathrm{CD}$ & $1.15 \mathrm{~F}$ & $0.86 \mathrm{DE}$ & $3.57 \mathrm{E}$ & $1.33 \mathrm{E}$ & $0.78 \mathrm{DE}$ \\
\hline AsA2 & $4.20 \mathrm{BCD}$ & $1.32 \mathrm{D}$ & $0.96 \mathrm{~A}$ & $3.97 \mathrm{C}$ & $1.52 \mathrm{C}$ & $0.90 \mathrm{~A}$ \\
\hline AsA3 & $4.55 \mathrm{~A}$ & $1.21 \mathrm{E}$ & $0.92 \mathrm{~B}$ & $4.00 \mathrm{C}$ & $1.41 \mathrm{D}$ & $0.84 \mathrm{~B}$ \\
\hline
\end{tabular}

${ }^{\mathrm{Z}}$ :Mo 1,2,3= 2,4,6 (g/l), respectively; Cyto 1,2,3= 50,100,200 (ppm), respectively; AsA $1,2,3=50,100,200(\mathrm{~g} / \mathrm{l})$, respectively.

Values within the same column followed by the same letters are not significantly different at 5\% level Duncan's multiple range test.

Egypt. J. Hort. Vol. 42, No.1 (2015) 


\section{Chlorophyll and carbohydrates}

The results presented in Table 5 indicate that foliar application with benzyladenine at $100 \mathrm{ppm}$ gave the best results in leaf chlorophyll content. Also, the same concentration from ascorbic acid was quiet better. On the other hand, chlorophyll continue to increase as moringa extract concentration also increased lead us to a conclusion that higher concentration (than which were used in this study) may be more effective. In this regard, some investigators reported that moringa leaf extract increased chlorophyll (a) pigment in tomato (Azra et al., 2014). Ibrahim et al. (2007) detected an increase in photosynthetic pigments under the influence of benzyl adenine at the rate of $100 \mathrm{ppm}$.

This increase in chlorophyll was concomitant with substantial accumulation of carbohydrates, as the chloroplasts are the factories of carbohydrates production in plants. Consequently, the carbohydrate concentration increased with the above mentioned application. The increase in carbohydrate with the best application used (benzyladenine at $100 \mathrm{ppm}$ ) was $34.3 \%$ and $30.9 \%$ over the control in first and second seasons, respectively. It was found that, total carbohydrate increment in vicia faba plants was detected by using 100, 200 or $400 \mathrm{mg} /$ litre ascorbic acid (El Bassiouny et al., 2005). In addition, benzyl adenine and other bioregulators caused significant increase $(59.94 \%)$ in total carbohydrate percentage of the produced vicia faba seeds resulted from the treated plants (Ibrahim et al., 2007).

TABLE 5. Chlorophyll and carbohydrates content of squash leaves as affected by foliar application with different concentrations of moringa leaves extract (Mo), cytokinene (cyto) and ascorbic acid (Asc) in seasons 2013 \& 2014.

\begin{tabular}{|c|c|c|c|c|}
\hline \multirow{2}{*}{ Character } & \multicolumn{2}{|c|}{$\mathbf{1}^{\text {st }}$ Season } & \multicolumn{2}{c|}{$\mathbf{2}^{\text {nd }}$ Season } \\
\cline { 2 - 5 } & $\begin{array}{c}\text { Chlorophyll } \\
\text { (SPAD) }\end{array}$ & $\begin{array}{c}\text { Carbohydrates } \\
(\%)\end{array}$ & $\begin{array}{c}\text { Chlorophyll } \\
\text { (SPAD) }\end{array}$ & $\begin{array}{c}\text { Carbohydrates } \\
(\%)\end{array}$ \\
\hline CONT. & $41.20 \mathrm{~F}$ & $32.53 \mathrm{G}$ & $42.30 \mathrm{~F}$ & $33.19 \mathrm{~F}$ \\
\hline Mo1 & $42.50 \mathrm{E}$ & $39.27 \mathrm{CD}$ & $43.06 \mathrm{E}$ & $39.96 \mathrm{CD}$ \\
\hline Mo2 & $43.90 \mathrm{D}$ & $42.11 \mathrm{AB}$ & $43.80 \mathrm{D}$ & $41.87 \mathrm{ABC}$ \\
\hline Mo3 & $45.10 \mathrm{C}$ & $42.27 \mathrm{AB}$ & $44.90 \mathrm{C}$ & $42.31 \mathrm{AB}$ \\
\hline Cyto1 & $43.80 \mathrm{D}$ & $35.81 \mathrm{~F}$ & $44.03 \mathrm{D}$ & $39.04 \mathrm{DE}$ \\
\hline Cyto2 & $49.60 \mathrm{~A}$ & $43.68 \mathrm{~A}$ & $51.20 \mathrm{~A}$ & $43.46 \mathrm{~A}$ \\
\hline Cyto3 & $45.80 \mathrm{~B}$ & $40.66 \mathrm{BCD}$ & $46.50 \mathrm{~B}$ & $41.18 \mathrm{BC}$ \\
\hline AsA1 & $43.00 \mathrm{E}$ & $37.11 \mathrm{EF}$ & $43.90 \mathrm{D}$ & $37.64 \mathrm{E}$ \\
\hline AsA2 & $45.90 \mathrm{~B}$ & $41.16 \mathrm{BC}$ & $45.20 \mathrm{C}$ & $41.38 \mathrm{BC}$ \\
\hline AsA3 & $44.10 \mathrm{D}$ & $38.65 \mathrm{DE}$ & $44.90 \mathrm{C}$ & $39.11 \mathrm{DE}$ \\
\hline
\end{tabular}

$\mathrm{Z}$ :Mo 1,2,3= 2,4,6 (g/l), respectively; Cyto 1,2,3=50,100,200 (ppm), respectively; AsA $1,2,3=$ $50,100,200(\mathrm{~g} / \mathrm{l})$, respectively.

Values within the same column followed by the same letters are not significantly different at $5 \%$ level Duncan's multiple range test. 


\section{Conclusion}

Moringa is a highly valued plant and has attained massive awareness due to its leaf composition being rich in cytokines, vitamins, antioxidants and other growth regulators. In this study, leaf extract of moringa at three levels, i.e. 2, 4, 6 $\mathrm{g} / \mathrm{L}$ had been applied as foliar spray on squash plants. The results indicated that, the growth and seed yield of squash were generally improved as a result of moringa application in a magnitude that was directly proportional to the concentration used. Thus we can assume that higher concentration may be more effective. Using Benzyle adenine (cytokine) or ascorbic acid were also effective by using them as foliar spray which improved all studded growth attributes of squash, especially at $100 \mathrm{ppm}$ concentration.

\section{References}

A.O.A.C. (2003) "Official Methods of Analysis", Association of Official Analytical Chemists. $17^{\text {th }}$ ed., $2^{\text {nd }}$ revision. Gaithersburg, MD, USA.

Bamishaiye, E.I., Olayemi, F.F., Awagu, E.F. and Bamshaiye, O.M. (2011) Proximate and phytochemical composition of Moringa oleifera leaves at three stages of maturation. Advance Journal of Food Science and Technology, 3 (4), 233-237.

Batlang, U., Emongor, V.E. and Pule-Meulenburg (2006) Effect of benzyladenine plus gibberellins and gibberellic acid on yield and yield components of cucumber (Cucumis sativus L. cv. Tempo). Journal of Agronomy, 5 (3), 418-423.

Bauernfeind, J.C. (2009) Ascorbic Acid Technology in Agricultural, Pharmaceutical, Food, and Industrial Applications. Advances in Chemistry. vol 200. Chapter (Book) Ascorbic Acid: Chemistry, Metabolism and Uses. Chapter 20, 395-497.

Borkowska, B. (1997) Cytokininy. In: L.S. Jankiewicz (Ed.). Regulatory wzrostu i rozwoju roślin. Wyd. Nauk. PWN, Warszawa: 60-71.

Chaudhary, N. and Agrawal, S.B. (2014) Cultivar specific variations in morphological and biochemical characteristics of mung bean due to foliar spray of ascorbic acid under elevated ozone. Acta Physiologiae Plantarum, 36 (7), 1793-1803.

Culver1, M., Fanuel1, T. and Chiteka1, A.Z. (2012) Effect of moringa extract on growth and yield of Tomato. Greener Journal of Agricultural, 2 (5), 207-211.

Duke, J.A. (1992) Handbook of Phytochemical Constituents of GRAS Herbs and Other Economic Plants. Boca Raton, Florida. CRC Press.

Duncan, D.B. (1955) Multiple Range and Multiple F-Tests. Biometrics, 11, 1-42.

El Kramany, M.F., Bahr, A. A., Mohamed, M.F. and Kabesh, M.O. (2007) Utilization of bio-Fertilizers in field crops production 16-Groundnut Yield, its Components and Seeds Content as Affected by Partial Replacement of Chemical Fertilizers by BioOrganic Fertilizers. Journal of Applied Sciences Research, 3 (1), 25-29. 
El-Bassiouny, H.M.S., Gobarah, M.E. and Ramadan, A.A. (2005) Effect of antioxidants on growth, yield and favism causative agents in seeds of Vicia faba L. plants grown under reclaimed sandy soil. Journal of Agronomy, 4 (4), 281-287.

El-Hifny, I.M.M. and El-Sayed, M.A.M. (2011) Response of sweet pepper plant growth and productivity to application of ascorbic acid and biofertilizers under saline conditions. Australian Journal of Basic and Applied Sciences, 5 (6), 1273-1283.

El-Tohamy, W.A., El-Abagy, H.M. and El-Greadly, N.H.M. (2008) Studies on the effect of putrescine, yeast and vitamin $\mathrm{C}$ on growth, yield and physiological responses of eggplant (Solanum melongena L.) under sandy soil conditions. Australian Journal of Basic and Applied Sciences, 2 (2), 296-300.

Emongor, V.E. (2007) Effect of Accel on growth, yield and yield components of snap bean (Phaseolus vulgaris). African Crop Science Conference Proceedings. African Crop Science Society, 8, 457-462.

Fuglie, L.J. (2000) New uses of moringa studied in Nicaragua: ECHO's Technical Network Site networking global hunger solutions. ECHO, Nicaragua.

Herbert, D., Phipps, P.J. and Strange, R.E. (1971) Chemical analysis of microbial cells. Methods in Microbiology, 5 (B), 209-344.

Ibrahim, M.E., Bekheta, M.A., El-Moursi, A. and Gaafar, N.A. (2007) Improvement of growth and seed yield quality of Vicia faba L. plants as affected by application of some bioregulators. Australian Journal of Basic and Applied Sciences, 1 (4), 657-666.

Jyoti, U. and Malik, C.P. (2013) Putrescine and ascorbic acid mediated enhancement in growth and antioxidant status of Eruca sativa varieties. Journal of Biotechnology, 2 (4), 53-64.

Naeem, M., Bhatti, I., Ahmad, R.H. and Ashraf, M.Y. (2004) Effect of some growth hormones $\left(\mathrm{GA}_{3}\right.$, IAA and kinetin) on the morphology and early or delayed initiation of bud of lentil (Lens culinaris medik). Pakistan Journal of Botany, 36 (4), 801-809.

Phiri, C. and Mbewe, D.N. (2010) Influence of Moringa oleifera leaf extracts on germination and seedling survival of three common legumes. International Journal of Agriculture and Biology, 12 (2), 315-317.

Salama, Z.A., El-Zanaty, A.A., Abou El-Nour, El-Fouly, M.M. and Gaafar, A.A. (2014) Ascorbic acid foliar spray counteracting effect of salinity on growth, nutrients concentrations, photosynthesis, antioxidant activities and lipid peroxidation of bean (Phaseulus vulgaris L.) cultivars. American Journal of Agricultural and Biological Science, 9 (3), 384-393.

Snedecor, G.W. and Cochran, W.G. (1980) "Statistical Methods", $7^{\text {th }}$ ed., Iowa State University Press, Ames, Iowa.

Sowley, E.N.K., Kankam, F. and Ado*mako, J. (2014) Management of root-knot nematode (Meloidogyne spp.) on sweet pepper (Capsicum annuum L.) with moringa (Moringa oleifera Lam.) leaf powder. Archives of Phytopathology and Plant Protection, 47 (13),1531-1538. 
Suza, W.P., Avila, C.A., Carruthers, K., Kulkarni, S., Goggin, F.L. and Lorence, A. (2010) Exploring the impact of wounding and jasmonates on ascorbate metabolism. (Special Issue: Antioxidants and redox regulation in plants.). Plant Physiology and Biochemistry. 48 (5), 337-350.

Yasmeen, A., Nouman W., Basra, S.M.A., Abdul Wahid, Hafeez-ur-Rehman, Hussain N, Afzal I. (2014) Morphological and physiological response of tomato (Solanum lycopersicum L.) to natural and synthetic cytokinin sources: a comparative study. Acta Physiologiae Plantarum, 36 (12), 3147-3155.

(Received 13/10/2014; accepted $12 / 2 / 2015)$

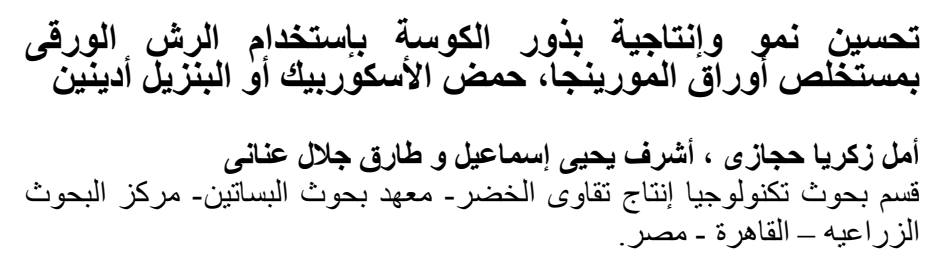

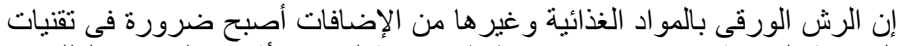







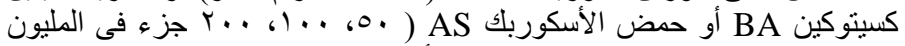

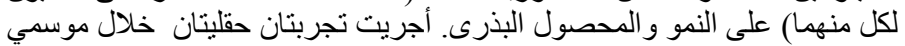

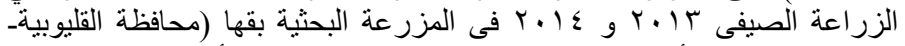

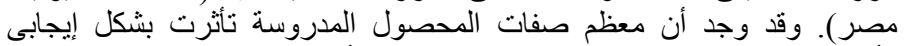

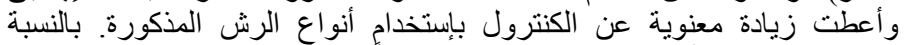

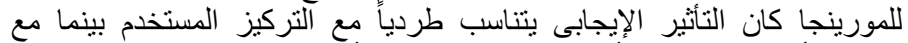

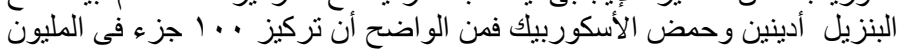



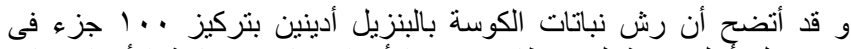

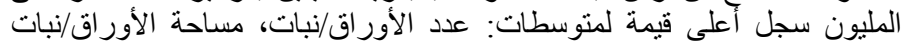

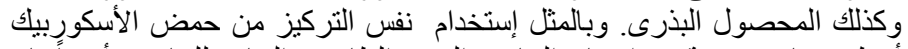

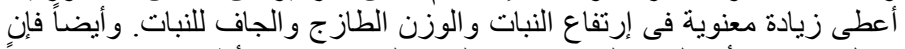

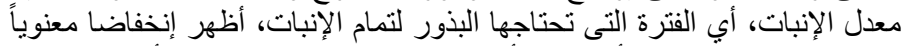

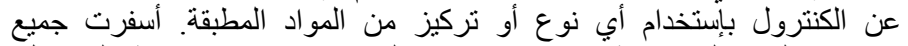

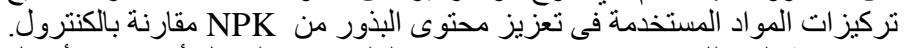




الكربو هيدر ات. ترياد.

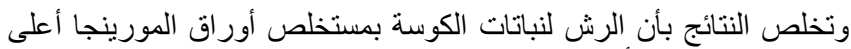

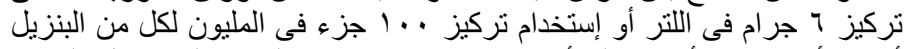

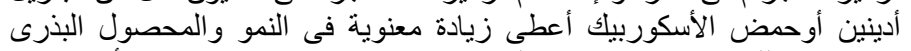

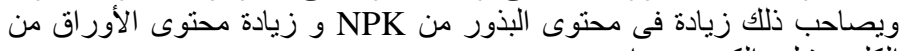
الكلوروفيل و الكربو هيدر ات فيات.

Egypt. J. Hort. Vol. 42, No.1 (2015) 